PHYSICAL REVIEW D 90, 066004 (2014)

\title{
Entanglement entropy and D1-D5 geometries
}

\author{
Stefano Giusto ${ }^{1,2, *}$ and Rodolfo Russo ${ }^{3, \dagger}$ \\ ${ }^{1}$ Dipartimento di Fisica ed Astronomia "Galileo Galilei," Università di Padova, \\ Via Marzolo 8, 35131 Padova, Italy \\ ${ }^{2}$ I.N.F.N. Sezione di Padova, Via Marzolo 8, 35131 Padova, Italy \\ ${ }^{3}$ Centre for Research in String Theory, School of Physics and Astronomy, \\ Queen Mary University of London, Mile End Road, \\ London E1 4NS, United Kingdom \\ (Received 17 June 2014; published 4 September 2014)
}

\begin{abstract}
In conformal field theories (CFTs) with a gravitational anti-de Sitter (AdS) dual it is possible to calculate the entanglement entropy of a region $A$ holographically by using the Ryu-Takayanagi formula. In this work we consider systems that are in a pure state that is not the vacuum. We study in particular the two-dimensional conformal field theory dual to type IIB string theory on $\mathrm{AdS}_{3} \times S^{3} \times T^{4}$ and focus on the 1/4-BPS states described holographically by the two-charge microstate geometries. We discuss a general prescription for the calculation of the entanglement entropy in these geometries that are asymptotically $\mathrm{AdS}_{3} \times S^{3}$. In particular we study analytically the perturbative expansion for a single, short interval: we show that the first nontrivial terms in this expansion are consistent with the expected CFT structure and with previous results on the vacuum expectation values of chiral primary operators for the 1/4-BPS configurations.
\end{abstract}

DOI: 10.1103/PhysRevD.90.066004

PACS numbers: 11.25.Tq, 04.50.Gh, 11.10.Kk, 03.67.Mn

\section{INTRODUCTION}

Entanglement entropies in quantum field theory have been at the center of intense study in the last few years, in particular in the case of conformal field theories (CFTs) that admit a dual gravitational description. In $1+1 \mathrm{D} \mathrm{CFTs}$, which will be the focus of this work, Rényi and von Neumann's entanglement entropies (EEs) can be calculated in terms of correlators among local operators by using the replica trick [1]. On the gravitational side, von Neumann's EE can be computed via the Ryu-Takayanagi (RT) formula [2] and the generalization to Rényi's case was discussed in [3-5]. A general argument explaining the RT formula has been recently given in [6]. Most of the past work focused on density matrices $\rho_{A}$ obtained starting either from the $S L(2, \mathbb{C})$ invariant ground state [(dual to anti-de Sitter (AdS)] or from the thermal state (dual to the BanadosTeitelboim-Zanelli (BTZ) black hole [7]) and tracing the degrees of freedom outside the space region $A$. When $A$ is an interval, the EE is given directly in terms of the central charge $c$ and does not depend on other details of the CFT. Things are more complicated if the space region $A$ is made of several disconnected intervals $[8,9]$ and already the case of two disjoint intervals $[10,11]$ provides a good testing ground to study nonuniversal quantities.

In this work we focus on a different setup which also yields theory specific results: we study the EE for a density matrix obtained from a pure state $|s\rangle$ that is not the

\footnotetext{
*stefano.giusto@pd.infn.it

†rrusso@qmul.ac.uk
}

$S L(2, \mathbb{C})$ invariant vacuum. On the holographic side, it was first suggested in [7] that the analysis of the EE in microstate geometries that are asymptotically $\mathrm{AdS}_{3}$ represents a first step to understand microscopically the result for the extremal BTZ black hole. From the CFT point of view a similar problem has been recently analyzed in [12] and [13-15]. While the latter references focus on a timedependent situation, we will focus, as in [12], on a density matrix obtained from an eigenstate $|s\rangle$ of the CFT Hamiltonian. This reference assumes that $|s\rangle$ is a small perturbation of the vacuum state. Since we aim to provide also a gravitational description of our analysis we focus on a $1+1 \mathrm{D}$ CFT that has a well-known string dual. The states we will be considering induce a macroscopic backreaction on the dual geometry and thus we need to consider the $\mathrm{EE}$ in a background that is not just $\mathrm{AdS}_{3}$ plus a small perturbation. We argue that this requires a generalization of the standard RT formula and check explicitly in some cases that the holographic results match the CFT expectations.

In particular we will focus on the superconformal field theory with $(4,4)$ supercharges and central charge $c=6 n_{1} n_{5}$, whose dual gravitational description is given in terms of type IIB string theory compactified on $S^{1} \times T^{4}$ with $n_{1}$ D1-branes and $n_{5}$ D5-branes wrapped on the compact space (the radius $R$ of the $S^{1}$ is much bigger than the string sized $T^{4}$ and all the branes wrap this circle). The gravitational description is appropriate for large charges $n_{1}, n_{5} \gg 1$ and for particular values of the moduli of the CFT. However it is convenient also to keep in mind a free field representation of the CFT with four bosonic and four 
fermionic fields whose target space is $\left(T^{4}\right)^{n_{1} n_{5}} / S_{n_{1} n_{5}}$. This particular AdS/CFT duality has been thoroughly studied also because of its application to black hole physics in five dimensions: the Strominger-Vafa black hole [16] counts 1/8-BPS states in this CFT and, in general, this setup can be used to address questions about the gravitational nature of each pure semiclassical state, a topic which is at the center of the so-called fuzzball program ${ }^{1}$ [23].

Our main goal is to study the EE for a single interval in the BPS states preserving $1 / 4$ of the 32 supercharges of the type IIB theory. From the CFT point of view this means that we consider only the ground states in the Ramond-Ramond sector (i.e. the sector where the fermions have periodic boundary conditions). Of course these are eigenstates of the CFT Hamiltonian, with zero energy, so we are dealing with a stationary (but nonstatic) configuration. In particular we will focus on semiclassical states, which are dual to smooth geometries on the bulk side of the AdS/CFT correspondence. The general form of these solutions is known [24-26] and we use it to compute holographically the EE of an interval. While the calculation can be set up in general, in order to give explicit results we focus on the limit where the size of the interval is small with respect to the $S^{1}$ where the CFT is defined (which coincides with the large $S^{1}$ in the string compactification). This limit allows for analytic calculations both on the gravity and the CFT sides, and, in the case of two intervals in the ground states, it was studied in $[3,10,11]$. The first subleading term in this expansion is sufficient to show that there exists a state specific contribution beyond the leading universal result.

The outline of this paper is as follows. In Sec. II we first describe the general prescription for calculating the $\mathrm{EE}$ in a stationary geometry that is asymptotically $\operatorname{AdS} \times \mathcal{M}$, where $\mathcal{M}$ is a compact space. As the geometries we consider are generically nonstatic, our prescription generalizes the covariant Hubeny-Rangamani-Takayanagi (HRT) [27] formalism for the holographic computation of the EE. Then we focus on the 1/4 BPS geometries of [24-26], which correspond to the Ramond-Ramond ground states of the dual CFT. The result for the EE of a single interval is given in terms of an integral which includes the compact space. In Sec. III we discuss in detail the short interval limit up to the first nonuniversal terms. In Sec. IV we reinterpret the gravity result in terms of the underlying CFT. The quantity under analysis is nonprotected and so it is not possible to use directly the free orbifold description. However, the short interval expansion can be written in a general way as a sum of terms related to the different operators of the CFT. Operators with a large conformal dimension give a negligible contribution to the $\mathrm{EE}$ in this limit and so we can limit ourselves to a finite number of

\footnotetext{
${ }^{1}$ Recent reviews on the subject are [17-19], and a discussion of some general implications of this approach for the physics of black holes can be found in [20-22].
}

operators with a small dimension. In the strong coupling limit, where the gravity approximation is valid, only the BPS operators have a finite dimension. In this section, we indeed show that the gravity result discussed previously matches the general CFT expression for the short interval expansion where only the contribution of the BPS operator is included. Further comments explaining why it is possible to make this comparison at a precise, quantitative level can be found after Eq. (4.9). To the best of our knowledge, this result represents the first nontrivial check of the RT formula (or more precisely of its six-dimensional extension) in a situation where the EE has also nonuniversal contributions.

\section{HOLOGRAPHIC ENTANGLEMENT ENTROPY}

In theories that admit a holographic dual, the EE can be computed via the RT formula [2]. In its simplest form, the formula applies to theories whose gravity dual is classical Einstein gravity $^{2}$ (eventually plus matter) and to states dual to static classical spacetimes that tend asymptotically to $\operatorname{AdS}_{d+1}$. For $d=2$, the EE of a one-dimensional spatial region $A$ is given by

$$
S_{A}=\frac{\operatorname{area}\left(\gamma_{A}\right)}{4 G_{N}},
$$

where $\gamma_{A}$ is the curve of minimal length homologous to $A$, in the space slice of the bulk containing $A$, and $G_{N}$ is the Newton's constant of the three-dimensional theory.

We aim to apply the RT formalism to compute entanglement entropies in states of the D1-D5 black hole. These states can be identified with the Ramond-Ramond (RR) ground states of a two-dimensional CFT that we will denote as the D1-D5 CFT (for a review see [31]). The gravitational duals of these states are described semiclassically by tendimensional supergravity solutions that, in the decoupling limit, are asymptotically $\mathrm{AdS}_{3} \times S^{3} \times T^{4}$. As the $T^{4}$ is taken to have string size, the geometries are smeared on the $T^{4}$ and can be equivalently described by six-dimensional solutions, with Einstein metric $d s_{6}^{2}$. Generic microstates depend however nontrivially on the $S^{3}$ directions, and there is no canonical way to reduce them to three-dimensional asymptotically $\mathrm{AdS}_{3}$ solutions. We thus need a generalization of the RT formula (2.1) that applies to six-dimensional spacetimes asymptotic to $\mathrm{AdS}_{3} \times S^{3}$. Given a one-dimensional spatial region $A$, we propose that its $\mathrm{EE}$ in a D1-D5 microstate is given by

$$
S_{A}=\frac{\operatorname{area}\left(\Gamma_{A}\right)}{4 G_{N}^{\prime}}
$$

where $\Gamma_{A}$ is the four-dimensional minimal-area surface of the six-dimensional geometry at constant time that at the

\footnotetext{
${ }^{2}$ The generalization to holographic theories with higher curvature corrections has been worked out in [28,29]; see also [30].
} 
$\mathrm{AdS}_{3}$ boundary reduces to $\partial A \times S^{3}$ and in the bulk has the product structure defined below; $G_{N}^{\prime}$ is the six-dimensional Newton's constant. In order to provide a precise definition of the class of four-dimensional manifolds to which $\Gamma_{A}$ belongs, one needs to give meaning to the split of the sixdimensional space into an $\mathrm{AdS}_{3}$ and an $S^{3}$ part or in other words to introduce an almost product structure. While this split can be unambiguously defined at the boundary of the space, where the geometry reduces to $\mathrm{AdS}_{3} \times S^{3}$, there are various inequivalent ways to extend it in the interior of the bulk. An almost product structure can be defined by choosing a system of coordinates $x^{I}=\left(x^{\mu}, x^{\alpha}\right)$ (with $I=1, \ldots 6, \quad \mu=1, \ldots, 3, \quad \alpha=1, \ldots, 3)$, where, at the boundary, $x^{\mu}$ and $x^{\alpha}$ are coordinates in $\mathrm{AdS}_{3}$ and $S^{3}$. These coordinates are extended in the bulk in such a way that the six-dimensional Einstein metric $G_{I J}$ satisfies the de Donder-Lorentz gauge ${ }^{3}$

$$
\nabla^{\alpha} \hat{G}_{\alpha \beta}=\nabla^{\alpha} G_{\alpha \mu}=0,
$$

where covariant derivatives are defined with respect to the round $S^{3}$ metric and $\hat{G}_{\alpha \beta}$ is the traceless part of $G_{\alpha \beta}$. Using these coordinates, the six-dimensional Einstein metric can be written in the form

$$
\begin{aligned}
d s_{6}^{2} & =G_{I J} d x^{I} d x^{J} \\
& =g_{\mu \nu} d x^{\mu} d x^{\nu}+G_{\alpha \beta}\left(d x^{\alpha}+A_{\mu}^{\alpha} d x^{\mu}\right)\left(d x^{\beta}+A_{\nu}^{\beta} d x^{\nu}\right),
\end{aligned}
$$

which defines the split of the six-dimensional metric into deformed $\mathrm{AdS}_{3}$ and $S^{3}$ parts indicated as $g_{\mu \nu}$ and $G_{\alpha \beta}$ respectively. As usual in Kaluza-Klein (KK) reductions, this split is invariant under reparametrizations of the compact space $\left(x^{\alpha} \rightarrow x^{\alpha}\left(x^{\mu}, x^{\beta}\right)\right)$, but is not invariant under $x^{\alpha}$-dependent changes of the coordinates $x^{\mu}$ $\left(x^{\mu} \rightarrow x^{\mu}\left(x^{\nu}, x^{\alpha}\right)\right)$; it is precisely this arbitrariness that is fixed by the gauge condition (2.3). So the prescription we propose is to minimize the functional (2.2) over the class of 4-manifolds that is invariant under the almost product structure induced by the coordinate split (2.4). These manifolds can be parametrized as $x^{I}\left(\lambda, x^{\alpha}\right)=\left(x^{\mu}(\lambda), x^{\alpha}\right)$, where $x^{0}(\lambda)=$ const. when $\Gamma_{A}$ lies in a constant time slice. The metric induced on the 4-manifold $\Gamma_{A}$ is

$$
d s_{*}^{2}=g_{\mu \nu} \dot{x}^{\mu} \dot{x}^{\nu} d \lambda^{2}+G_{\alpha \beta}\left(d x^{\alpha}+A_{\mu}^{\alpha} \dot{x}^{\mu} d \lambda\right)\left(d x^{\beta}+A_{\nu}^{\beta} \dot{x}^{\nu} d \lambda\right),
$$

\footnotetext{
${ }^{3}$ The de Donder-Lorentz gauge is the one commonly employed when reducing on compact spaces [32], and it seems natural in the AdS/CFT context, because it was shown in [33] that it reproduces the results of a gauge-invariant $\mathrm{KK}$ reduction procedure.
}

its determinant is

$$
\operatorname{det}\left(g_{*}\right)=g_{\mu \nu} \dot{x}^{\mu} \dot{x}^{\nu} \operatorname{det}\left(G_{\alpha \beta}\right),
$$

and the area of the 4-manifold is

$$
\begin{aligned}
\operatorname{area}\left(\Gamma_{A}\right) & =\int d \lambda d^{3} x^{\alpha} \sqrt{\operatorname{det}\left(G_{\alpha \beta}\right)} \sqrt{g_{\mu \nu} \dot{x}^{\mu} \dot{x}^{\nu}} \\
& \equiv \int d \lambda d^{3} x^{\alpha} \sqrt{g_{\mu \nu}^{E} \dot{x}^{\mu} \dot{x}^{\nu}},
\end{aligned}
$$

where we have defined

$$
g_{\mu \nu}^{E}=g_{\mu \nu} \operatorname{det}\left(G_{\alpha \beta}\right)
$$

For generic microstates $g_{\mu \nu}^{E}$ depends nontrivially on the $S^{3}$ coordinates $x^{\alpha}$. When instead $g_{\mu \nu}^{E}$ in (2.8) is independent of $x^{\alpha}$, it is just the Einstein metric of the three-dimensional theory reduced on $S^{3}$, and the prescription (2.2) reduces to the RT formula (2.1) for the asymptotically $\mathrm{AdS}_{3}$ metric $g_{\mu \nu}^{E}$.

Generic microstates are, moreover, associated with stationary but nonstatic geometries. It was shown in [27] that for nonstatic geometries the RT prescription has to be generalized by relaxing the constraint that the class of manifolds over which one minimizes the area functional lies in a constant time slice. In this more general setting, minimal surfaces might no longer exist, and one should look instead for extremal surfaces. We will denote this covariant generalization of the RT prescription as the HRT prescription. The HRT formalism can be generalized to spacetimes asymptotic to $\mathrm{AdS}_{3} \times S^{3}$ along the same lines outlined above: the covariant six-dimensional prescription is to find the extrema of the area functional (2.7) over manifolds $\Gamma_{A}$ that are invariant under the almost product structure previously defined, without imposing any restriction on $x^{0}(\lambda)$ in the bulk.

\section{A. Solution of the geodesic problem for a single interval}

Let us now work out the equations satisfied by extremal surfaces in a general nonstatic geometry. For the purpose of extremizing the area functional (2.7) with respect to the functions $x^{\mu}(\lambda)$, the $S^{3}$ coordinates $x^{\alpha}$ play the role of external parameters, and explicit dependence on $x^{\alpha}$ will be suppressed in the following: it is understood that everything is computed at some fixed value of $x^{\alpha}$, over which one integrates at the end. As usual, it is convenient to parametrize $x^{\mu}(\lambda)$ in terms of the "proper time" parameter $\tau$, satisfying

$$
g_{\mu \nu}^{E} \dot{x}^{\mu} \dot{x}^{\nu}=1
$$

In this parametrization, extrema of the area functional satisfy 


$$
\frac{d}{d \tau}\left(g_{\mu \nu}^{E} \dot{x}^{\mu}\right)=\frac{1}{2} \partial_{\nu} g_{\mu \lambda}^{E} \dot{x}^{\mu} \dot{x}^{\lambda} .
$$

Two-charge microstate geometries do not depend on the angular coordinate of $\mathrm{AdS}_{3}$, which is identified with the spatial coordinate of the CFT and will be denoted by $y$, but only on the $\mathrm{AdS}_{3}$ radial coordinate $r$ (apart from $x^{\alpha}$ ). Moreover we will assume for simplicity the gauge $g_{r t}^{E}=g_{r y}^{E}=0$; as we will see it is straightforward to satisfy this condition at the leading order in the large $r$ expansion. The relevant metric components are then $g_{r r}^{E}(r)$ and $g_{m n}^{E}(r)$ where we denote by $m, n$ indices that take values $t, y$. The components $\nu=n=t, y$ of the extremality equations (2.10) give

$$
\frac{d}{d \tau}\left(g_{m n}^{E}(r) \dot{x}^{m}\right)=0 \Rightarrow \dot{x}^{m}=g_{E}^{m n}(r) \kappa_{n},
$$

where $g_{E}^{m n}$ is the inverse of $g_{m n}^{E}$ and $\kappa_{n}$ are constants (that might depend on $x^{\alpha}$ ). The constraint (2.9) implies

$$
\begin{gathered}
g_{r r}^{E}(r) \dot{r}^{2}+g_{m n}^{E}(r) \dot{x}^{m} \dot{x}^{n}=1 \\
\Rightarrow \dot{r}^{2}=\frac{1-g_{E}^{m n}(r) \kappa_{m} \kappa_{n}}{g_{r r}^{E}(r)} .
\end{gathered}
$$

Equations (2.11), (2.12) determine $x^{\mu}(\tau)$ after specifying the boundary conditions which depend on the choice of the spatial region $A$. We will restrict to spatial regions made of a single interval of length $l$ at $t=\bar{t}$. The end points of the curve $x^{\mu}(\tau)$ at the boundary of $\operatorname{AdS}_{3}(r \rightarrow \infty)$ have to coincide with the boundaries of the interval $A$. Since the area of a 4-manifold that extends all the way to $r=\infty$ diverges, to obtain a finite result it is necessary to introduce an IR cutoff $r_{0}$ and replace the $\mathrm{AdS}_{3}$ boundary with the surface $r=r_{0}$. This explains the choice of the following boundary conditions:

$r\left(\tau_{1}\right)=r_{0}, \quad t\left(\tau_{1}\right)=\bar{t}, \quad y\left(\tau_{1}\right)=0 ;$

$r\left(\tau_{2}\right)=r_{0}, \quad t\left(\tau_{2}\right)=\bar{t}, \quad y\left(\tau_{2}\right)=l$.

Then

$$
\begin{aligned}
0 & =\int_{\tau_{1}}^{\tau_{2}} \dot{t} d \tau \\
& =2 \int_{r^{*}}^{r_{0}} \dot{\dot{t}} d r \\
& =2 \kappa_{m} \int_{r^{*}}^{r_{0}} d r g_{E}^{t m}(r) \sqrt{\frac{g_{r r}^{E}(r)}{1-g_{E}^{n p}(r) \kappa_{n} \kappa_{p}}},
\end{aligned}
$$

$$
\begin{aligned}
l & =\int_{\tau_{1}}^{\tau_{2}} \dot{y} d \tau \\
& =2 \int_{r^{*}}^{r_{0}} \frac{\dot{y}}{\dot{r}} d r \\
& =2 \kappa_{m} \int_{r^{*}}^{r_{0}} d r g_{E}^{y m}(r) \sqrt{\frac{g_{r r}^{E}(r)}{1-g_{E}^{n p}(r) \kappa_{n} \kappa_{p}}},
\end{aligned}
$$

where the turning point $r^{*}$ is the largest solution of

$$
g_{E}^{m n}\left(r^{*}\right) \kappa_{m} \kappa_{n}=1 .
$$

Inverting Eqs. (2.14)-(2.15) determines the parameters $\kappa_{m}$ in terms of the interval length $l$. These values of $\kappa_{m}$ can then be replaced in the expression for the area of the minimal submanifold $\Gamma_{A}$

$$
\begin{aligned}
\operatorname{area}\left(\Gamma_{A}\right) & =\int d^{3} x^{\alpha} \int_{\tau_{1}}^{\tau_{2}} d \tau \\
& =2 \int d^{3} x^{\alpha} \int_{r^{*}}^{r_{0}} \frac{1}{\dot{r}} d r \\
& =2 \int d^{3} x^{\alpha} \int_{r^{*}}^{r_{0}} d r \sqrt{\frac{g_{r r}^{E}(r)}{1-g_{E}^{m n}(r) \kappa_{m} \kappa_{n}}} .
\end{aligned}
$$

According to (2.2), the EE of the interval $A$ is then

$$
S_{A}=\frac{\operatorname{area}\left(\Gamma_{A}\right)}{4 G_{N}^{\prime}}=\frac{c}{6} \frac{\operatorname{area}\left(\Gamma_{A}\right)}{\operatorname{vol}\left(S_{b}^{3}\right) R_{\mathrm{AdS}}}=n_{1} n_{5} \frac{\operatorname{area}\left(\Gamma_{A}\right)}{\operatorname{vol}\left(S_{b}^{3}\right) R_{\mathrm{AdS}}},
$$

where $\operatorname{vol}\left(S_{b}^{3}\right)$ is the volume of the three-dimensional sphere at the boundary of AdS. We also used

$$
G_{N}^{\prime}=\operatorname{vol}\left(S_{b}^{3}\right) G_{N}, \quad c=\frac{3}{2} \frac{R_{\mathrm{AdS}}}{G_{N}}=6 n_{1} n_{5},
$$

with $n_{1}, n_{5}$ being the numbers of D1 and D5 branes and $R_{\text {AdS }}$ the radius of AdS.

\section{ENTANGLEMENT ENTROPY IN D1-D5 STATES FOR SMALL $l$}

The geometry of semiclassical D1-D5 states has been constructed in [26], whose conventions we will follow here. We will restrict for simplicity to the class of states that are invariant under rotations in the internal $T^{4}$ directions (for which $\mathcal{A}^{\alpha-}=0$, in the notation of [26]). The sixdimensional Einstein metric of these states is

$$
d s_{6}^{2}=f^{-1}\left[-(d t-A)^{2}+(d y-B)^{2}\right]+f d x^{i} d x^{i},
$$

where

$$
f \equiv\left(f_{1} f_{5}-\mathcal{A}^{2}\right)^{1 / 2},
$$


$x^{i}(i=1, \ldots, 4)$ are coordinates in $\mathbb{R}^{4}$, and $A \equiv A_{i} d x^{i}$, $B \equiv B_{i} d x^{i}$ are 1 -forms on $\mathbb{R}^{4}$ that satisfy $d B=-*_{4} d A$. $f_{1}, f_{5}, \mathcal{A}, A_{i}$ are harmonic functions on $\mathbb{R}^{4}$ whose explicit expressions are, for instance, given in Eqs. (2.12) and (2.5) of [26].

The simplest microstates are the ones with maximal or minimal values of the $S U(2)_{L} \times S U(2)_{R} R$-charges: $j= \pm n_{1} n_{5} / 2, \bar{j}= \pm n_{1} n_{5} / 2$. For example the geometry of the state with $j=\bar{j}=n_{1} n_{5} / 2$ is, in the decoupling limit,

$$
\begin{aligned}
d s_{6}^{2}= & -\frac{\hat{r}^{2}+a^{2}}{\sqrt{Q_{1} Q_{5}}} d t^{2}+\frac{\hat{r}^{2}}{\sqrt{Q_{1} Q_{5}}} d y^{2}+\sqrt{Q_{1} Q_{5}} \frac{d \hat{r}^{2}}{\hat{r}^{2}+a^{2}} \\
& +\sqrt{Q_{1} Q_{5}}\left(d \hat{\theta}^{2}+\cos ^{2} \hat{\theta} d \hat{\psi}^{2}+\sin ^{2} \hat{\theta} d \hat{\phi}^{2}\right),
\end{aligned}
$$

with

$$
\hat{\psi}=\psi-\frac{y}{R}, \quad \hat{\phi}=\phi-\frac{t}{R} .
$$

The parameter $a$ is related to the D1 and D5 charges, $Q_{1}$ and $Q_{5}$, and the radius $R$ of the $S^{1}$ direction $y$, by

$$
a=\frac{\sqrt{Q_{1} Q_{5}}}{R} .
$$

The coordinates $(\hat{r}, \hat{\theta})$ are mapped to polar coordinates of $\mathbb{R}^{4}(r, \theta)$ by

$$
r^{2}=\hat{r}^{2}+a^{2} \sin ^{2} \hat{\theta}, \quad \cos ^{2} \theta=\frac{\hat{r}^{2} \cos ^{2} \hat{\theta}}{\hat{r}^{2}+a^{2} \sin ^{2} \hat{\theta}} .
$$

It is immediate to check from (3.3) that these coordinates satisfy the de Donder-Lorentz gauge (2.3). Hence, in $\hat{r}, \hat{\theta}, \hat{\psi}, \hat{\phi}$ coordinates, it becomes explicit that the sixdimensional geometry of this particular microstate is simply $\mathrm{AdS}_{3} \times S^{3}$, and the three-dimensional geometry $g_{\mu \nu}^{E}$ reduced on $S^{3}$ is just global $\mathrm{AdS}_{3}$. According to the recipe (2.2), the $\mathrm{EE}$ of the interval $A=[0, l]$ computed in this state is the same as the one in the $S L(2, \mathbb{C})$-invariant vacuum:

$$
S_{A}=2 n_{1} n_{5} \log \left[\frac{2 r_{0}}{a} \sin \left(\frac{l}{2 R}\right)\right] .
$$

The metrics for generic microstates are too complicated to analytically carry out the holographic EE computation exactly. A limit which is amenable to analytic computations, both on the gravity and on the CFT side, is the short interval regime, in which $l$ is much smaller than the $S^{1}$ radius $R$. In this limit the extremal submanifold $\Gamma_{A}$ only probes the region of the geometry close to the boundary: hence only the large $r$ expansion of the geometry (3.1) is relevant in this approximation. We will consider just the first nontrivial correction in the $l$ expansion, and for this purpose one can approximate the metric coefficients as

$$
\begin{aligned}
f_{1} & \approx \frac{Q_{1}}{r^{2}}\left(1+\frac{f_{1 i}^{1}}{r} Y_{1}^{i}+\frac{f_{2 I}^{1}}{r^{2}} Y_{2}^{I}\right), \\
f_{5} & \approx \frac{Q_{5}}{r^{2}}\left(1+\frac{f_{1 i}^{5}}{r} Y_{1}^{i}+\frac{f_{2 I}^{5}}{r^{2}} Y_{2}^{I}\right), \\
\mathcal{A} & \approx \frac{\sqrt{Q_{1} Q_{5}} \mathcal{A}_{1 i}}{r^{3}} Y_{1}^{i}, \\
A & \approx \frac{\sqrt{Q_{1} Q_{5}}}{r^{2}}\left(a_{\alpha+} Y_{1}^{\alpha+}+a_{\alpha-} Y_{1}^{\alpha-}\right), \\
B & \approx \frac{\sqrt{Q_{1} Q_{5}}}{r^{2}}\left(a_{\alpha+} Y_{1}^{\alpha+}-a_{\alpha-} Y_{1}^{\alpha-}\right) .
\end{aligned}
$$

Here $f_{k I}^{1}, f_{k I}^{5}, \mathcal{A}_{1 i}, a_{\alpha \pm}$ are constants that can be computed once a specific two-charge microstate geometry is chosen. In the small $l$ expansion we are considering, we will only keep terms up to second order in $f_{1 i}^{1,5}, \mathcal{A}_{1 i}$, and $a_{\alpha \pm}$ and up to first order in $f_{2 I}^{1,5}$. It is always possible to pick coordinates in such a way that

$$
f_{1 i}^{1}+f_{1 i}^{5}=0,
$$

and we will take advantage of this gauge choice in the following. $Y_{k}^{I}$ are scalar spherical harmonics of degree $k$ on $S^{3}$. We will need in particular the harmonics of degree 1: the scalar $Y_{1}^{i}$, with $i=1, \ldots, 4$, and the vector harmonics $Y_{1}^{\alpha \pm}$, with $\alpha=1,2,3$, are

$$
Y_{1}^{i}=2 \frac{x^{i}}{r}, \quad Y_{1}^{\alpha+}=\frac{\eta_{i j}^{\alpha} d x^{i} x^{j}}{r^{2}}, \quad Y_{1}^{\alpha-}=\frac{\bar{\eta}_{i j}^{\alpha} d x^{i} x^{j}}{r^{2}},
$$

where $\eta_{i j}^{\alpha}=\delta_{\alpha i} \delta_{4 j}-\delta_{\alpha j} \delta_{4 i}+\varepsilon_{\alpha i j 4}, \bar{\eta}_{i j}^{\alpha}=\delta_{\alpha i} \delta_{4 j}-\delta_{\alpha j} \delta_{4 i}-\varepsilon_{\alpha i j 4}$ are the standard 't Hooft symbols. One can use either $Y_{1}^{\alpha+}$ or $Y_{1}^{\alpha-}$ to form a basis of 1 -forms on $S^{3}$, and moreover the round $S^{3}$ metric can be written as $d s_{3}^{2}=\sum_{\alpha} Y_{1}^{\alpha+} \otimes Y_{1}^{\alpha+}=$ $\sum_{\alpha} Y_{1}^{\alpha-} \otimes Y_{1}^{\alpha-}$. In order to rewrite the metric in the form (2.4), suitable to perform the reduction on $S^{3}$, it is convenient to express the 1-forms in one of the two bases, let us say $Y_{1}^{\alpha+}$. Hence we write

$$
Y_{1}^{\alpha-}=R_{\beta}^{\alpha} Y_{1}^{\beta+},
$$

where the coefficients $R_{\beta}^{\alpha}$ depend on the $S^{3}$ coordinates, and

$$
\begin{aligned}
& A \approx \frac{\sqrt{Q_{1} Q_{5}}}{r^{2}}\left(a_{\alpha+}+\tilde{a}_{\alpha-}\right) Y_{1}^{\alpha+}, \\
& B \approx \frac{\sqrt{Q_{1} Q_{5}}}{r^{2}}\left(a_{\alpha+}-\tilde{a}_{\alpha-}\right) Y_{1}^{\alpha+},
\end{aligned}
$$

with

$$
\tilde{a}_{\alpha-}=R_{\alpha}^{\beta} a_{\beta-} .
$$


The scalar and vector spherical harmonics in (3.10) satisfy

$$
\begin{aligned}
\left(Y_{1}^{\alpha+}\right)_{\gamma}\left(Y_{1}^{\beta+}\right)^{\gamma} & =\delta^{\alpha \beta}, & & \left(Y_{1}^{\alpha-}\right)_{\gamma}\left(Y_{1}^{\beta-}\right)^{\gamma}=\delta^{\alpha \beta}, \\
\frac{1}{2 \pi^{2}} \int_{S^{3}} d \Omega_{3} Y_{1}^{i} Y_{1}^{j} & =\delta^{i j}, & & \int_{S^{3}} d \Omega_{3} Y_{k}^{I}=0 \\
\int_{S^{3}} d \Omega_{3}\left(Y_{1}^{\alpha+}\right)_{\gamma}\left(Y_{1}^{\beta-}\right)^{\gamma} & =0, & &
\end{aligned}
$$

where the contraction over the $S^{3}$ indices $\gamma$ and the volume form $d \Omega_{3}$ are the ones associated with the round $S^{3}$ metric. This implies

$$
R_{\gamma}^{\alpha} R_{\gamma}^{\beta}=\delta^{\alpha \beta}, \quad \int_{S^{3}} R_{\beta}^{\alpha}=0 .
$$

The system of coordinates used in Eqs. (3.8) does not satisfy the de Donder-Lorentz gauge conditions (2.3) at the required order in the perturbative expansion. Before extracting the three-dimensional metrics $g_{\mu \nu}$ and $G_{\alpha \beta}$ from (2.4), one should thus change to coordinates satisfying the gauge (2.3). We have checked that, at our perturbative order, this procedure generates corrections to the $g_{\mu \nu}^{E}$ computed in the coordinates of (3.8) that are linear in the scalar harmonics of degree 2; hence these corrections vanish when integrated over $S^{3}$, thanks to the properties of spherical harmonics (3.14). For simplicity of exposition, we will thus continue working with the coordinates of (3.8).

At the required order in $1 / r$, the generic two-charge metric (3.1) can be recast in the form

$$
\begin{aligned}
d s_{6}^{2} \approx & f^{-1}\left[-\left(1+\frac{\left(a_{\alpha+}+\tilde{a}_{\alpha-}\right)^{2}}{r^{2}}\right) d t^{2}\right. \\
& \left.+\left(1-\frac{\left(a_{\alpha+}-\tilde{a}_{\alpha-}\right)^{2}}{r^{2}}\right) d y^{2}-2 \frac{\left(a_{\alpha+}\right)^{2}-\left(a_{\alpha-}\right)^{2}}{r^{2}} d t d y\right] \\
& +f\left(d r^{2}+r^{2} \hat{Y}_{1}^{\alpha+} \hat{Y}_{1}^{\alpha+}\right)-2 \frac{\sqrt{Q_{1} Q_{5}}}{r^{2}} a_{\alpha+} \tilde{a}_{\beta-} \hat{Y}_{1}^{\alpha+} \hat{Y}_{1}^{\beta+}
\end{aligned}
$$

with

$$
\hat{Y}_{1}^{\alpha+}=Y_{1}^{\alpha+}+\frac{a_{\alpha+}+\tilde{a}_{\alpha-}}{\sqrt{Q_{1} Q_{5}}} d t-\frac{a_{\alpha+}-\tilde{a}_{\alpha-}}{\sqrt{Q_{1} Q_{5}}} d y,
$$

and

$f \approx \frac{\sqrt{Q_{1} Q_{5}}}{r^{2}}\left(1-\frac{f_{1 i}^{1} f_{1 j}^{1}+\mathcal{A}_{1 i} \mathcal{A}_{1 j}}{2 r^{2}} Y_{1}^{i} Y_{1}^{j}+\frac{f_{2 I}^{1}+f_{2 I}^{5}}{2 r^{2}} Y_{2}^{I}\right)$.

The determinant of the $S^{3}$ metric $G_{\alpha \beta}$ at order $1 / r^{2}$ can be read off from (3.16)

$$
\operatorname{det}\left(G_{\alpha \beta}\right) \approx\left(f r^{2}\right)^{3} \operatorname{det} G_{S^{3}}\left(1-2 \frac{a_{\alpha+} a_{\beta-}}{r^{2}}\left(Y_{1}^{\alpha+}\right)_{\gamma}\left(Y_{1}^{\beta-}\right)^{\gamma}\right),
$$

where $\operatorname{det} G_{S^{3}}$ is the determinant of the metric for a round 3 -sphere of unit radius. In the same approximation the $\mathrm{AdS}_{3}$ metric defined in (2.8) is

$$
\begin{aligned}
d s_{E}^{2} \approx & \left(f r^{2}\right)^{3} \sin ^{2} \theta \cos ^{2} \theta\left(1-2 \frac{a_{\alpha+} a_{\beta-}}{r^{2}}\left(Y_{1}^{\alpha+}\right)_{\gamma}\left(Y_{1}^{\beta-}\right)^{\gamma}\right) \\
& \times\left\{f^{-1}\left[-\left(1+\frac{\left(a_{\alpha+}+\tilde{a}_{\alpha-}\right)^{2}}{r^{2}}\right) d t^{2}+\left(1-\frac{\left(a_{\alpha+}-\tilde{a}_{\alpha-}\right)^{2}}{r^{2}}\right) d y^{2}-2 \frac{\left(a_{\alpha+}\right)^{2}-\left(a_{\alpha-}\right)^{2}}{r^{2}} d t d y\right]+f d r^{2}\right\} \\
\equiv & \left(Q_{1} Q_{5}\right)^{3 / 2} \sin ^{2} \theta \cos ^{2} \theta\left[\frac{r^{2}}{\sqrt{Q_{1} Q_{5}}}\left[-\left(1+\delta g_{t t}^{E}\right) d t^{2}+\left(1+\delta g_{y y}^{E}\right) d y^{2}+2 \delta g_{t y}^{E} d t d y\right]+\frac{\sqrt{Q_{1} Q_{5}}}{r^{2}}\left(1+\delta g_{r r}^{E}\right) d r^{2}\right] .
\end{aligned}
$$

The metric $d s_{E}^{2}$ in general depends on the $S^{3}$ coordinates, but for the purpose of computing the area functional $\operatorname{area}\left(\Gamma_{A}\right)$ one can introduce a "reduced" $\mathrm{AdS}_{3}$ metric $d \hat{s}_{E}^{2}$, integrated over $S^{3}$, such that

$$
\frac{\operatorname{area}\left(\Gamma_{A}\right)}{4 G_{N}^{\prime}}=\frac{\int d \lambda d^{3} x^{\alpha} \sqrt{g_{\mu \nu}^{E} \dot{x}^{\mu} \dot{x}^{\nu}}}{4 G_{N}^{\prime}}=\frac{\int d \lambda \sqrt{\hat{g}_{\mu \nu}^{E} \dot{x}^{\mu} \dot{x}^{\nu}}}{4 G_{N}} .
$$

This reduced metric is given by

$$
\begin{aligned}
d \hat{s}_{E}^{2}= & \frac{r^{2}}{\sqrt{Q_{1} Q_{5}}}\left[-\left(1+\delta \hat{g}_{t t}^{E}\right) d t^{2}+\left(1+\delta \hat{g}_{y y}^{E}\right) d y^{2}\right. \\
& \left.+2 \delta \hat{g}_{t y}^{E} d t d y\right]+\frac{\sqrt{Q_{1} Q_{5}}}{r^{2}}\left(1+\delta \hat{g}_{r r}^{E}\right) d r^{2},
\end{aligned}
$$

where

$$
\delta \hat{g}_{\mu \nu}^{E}=\frac{1}{2 \pi^{2}} \int_{S^{3}} d \Omega_{3} \delta g_{\mu \nu}^{E} .
$$

Comparing with (3.20), and using (3.14) one finds (3.24) 


$$
\begin{aligned}
\delta \hat{g}_{t t}^{E} & =\frac{a_{+}^{2}+a_{-}^{2}-f_{1}^{2}-\mathcal{A}_{1}^{2}}{r^{2}}, \\
\delta \hat{g}_{y y}^{E} & =-\frac{a_{+}^{2}+a_{-}^{2}+f_{1}^{2}+\mathcal{A}_{1}^{2}}{r^{2}}, \\
\delta \hat{g}_{t y}^{E} & =-\frac{a_{+}^{2}-a_{-}^{2}}{r^{2}}, \quad \delta \hat{g}_{r r}^{E}=-2 \frac{f_{1}^{2}+\mathcal{A}_{1}^{2}}{r^{2}},
\end{aligned}
$$

where we introduced the condensed notation

$$
a_{ \pm}^{2} \equiv a_{\alpha \pm} a_{\alpha \pm}, \quad f_{1}^{2} \equiv f_{1 i}^{1} f_{1 i}^{1}, \quad \mathcal{A}_{1}^{2} \equiv \mathcal{A}_{1 i} \mathcal{A}_{1 i}
$$

Let us now apply the formalism of Sec. II A and determine the extremal curves for the reduced metric $\hat{g}_{\mu \nu}^{E}$. Note that $\hat{g}_{t y}^{E}$ is nontrivial and thus we should use the covariant prescription, without restricting to constant $x^{0}(\lambda)$. The EE, however, is invariant under changes of the orientation of the space $(y \rightarrow-y)$ and hence it will depend at least quadratically on $\hat{g}_{t y}^{E}{ }^{4}$ As in our case $\hat{g}_{t y}^{E}$ is already quadratic in $a_{\alpha \pm}$, its contributions to the EE will be quartic in $a_{\alpha \pm}$, and will be discarded at our perturbative order. We can thus simplify the computation and take $x^{0}(\lambda)=$ const and $\kappa_{t}=0$. Then Eq. (2.15) reads (at our level of approximation)

$$
\begin{aligned}
l & \approx 2 \kappa_{y}\left(Q_{1} Q_{5}\right)^{3 / 4} \int_{\hat{\kappa}}^{\infty} \frac{d r}{r^{2} \sqrt{r^{2}-\hat{\kappa}^{2}}}\left(1+\frac{a_{+}^{2}+a_{-}^{2}-f_{1}^{2}-\mathcal{A}_{1}^{2}}{2 r^{2}}\right) \\
& =2 \kappa_{y}\left(Q_{1} Q_{5}\right)^{3 / 4}\left(\frac{1}{\hat{\kappa}^{2}}+\frac{a_{+}^{2}+a_{-}^{2}-f_{1}^{2}-\mathcal{A}_{1}^{2}}{3 \hat{\kappa}^{4}}\right) \\
& \approx \frac{2 \sqrt{Q_{1} Q_{5}}}{\hat{\kappa}}\left(1-\frac{a_{+}^{2}+a_{-}^{2}+5\left(f_{1}^{2}+\mathcal{A}_{1}^{2}\right)}{6 \hat{\kappa}^{2}}\right)
\end{aligned}
$$

where in the last step we expanded for small $l$ and used the following expression for the turning point:

$$
\left(r^{*}\right)^{2} \equiv \hat{\kappa}^{2}=\sqrt{Q_{1} Q_{5}} \kappa_{y}^{2}+a_{+}^{2}+a_{-}^{2}+f_{1}^{2}+\mathcal{A}_{1}^{2} .
$$

Note that we sent $r_{0} \rightarrow \infty$ because the above integral is convergent. Equation (3.26) should be inverted to express $\hat{\kappa}$ in terms of $l$ :

$$
\hat{\kappa} \approx \frac{2 \sqrt{Q_{1} Q_{5}}}{l}\left(1-\frac{a_{+}^{2}+a_{-}^{2}+5\left(f_{1}^{2}+\mathcal{A}_{1}^{2}\right)}{24 Q_{1} Q_{5}} l^{2}\right) .
$$

We can now use Eqs. (2.17) and (2.18), and the fact that $R_{A d S}=\left(Q_{1} Q_{5}\right)^{1 / 4}$, to compute the EE for the interval $A=[0, l]$ in a generic two-charge state at order $l^{2}$ :

\footnotetext{
${ }^{4}$ This conclusion can also be verified directly from the equations of Sec. II A: it follows from (2.14) that $\kappa_{t}$ is of order $\hat{g}_{t y}^{E}$ and from (2.15) and (2.17) one sees that the EE receives contributions that are either proportional to $\kappa_{t}^{2}$ or to $\hat{g}_{t y}^{E} \kappa_{t}$.
}

$$
\begin{aligned}
S_{A} & \approx 2 n_{1} n_{5} \int_{\hat{\kappa}}^{r_{0}} \frac{d r}{\sqrt{r^{2}-\hat{\kappa}^{2}}}\left(1-\frac{a_{+}^{2}+a_{-}^{2}+3\left(f_{1}^{2}+\mathcal{A}_{1}^{2}\right)}{2 r^{2}}\right) \\
& =2 n_{1} n_{5}\left(\log \frac{r_{0}\left(1+\sqrt{1-\hat{\kappa} / r_{0}}\right)}{\hat{\kappa}}-\frac{a_{+}^{2}+a_{-}^{2}+3\left(f_{1}^{2}+\mathcal{A}_{1}^{2}\right)}{2 \hat{\kappa}^{2}}\right) \\
& \approx 2 n_{1} n_{5}\left(\log \frac{r_{0} l}{\sqrt{Q_{1} Q_{5}}}-\frac{a_{+}^{2}+a_{-}^{2}+2\left(f_{1}^{2}+\mathcal{A}_{1}^{2}\right)}{12 Q_{1} Q_{5}} l^{2}\right) .
\end{aligned}
$$

\section{COMPARING WITH THE CFT EXPECTATION}

In this section we show how to interpret the result in Eq. (3.29) from the CFT point of view. First we need to introduce a density matrix $\rho_{A}^{(s)}$ that is obtained by starting from a pure state $|s\rangle$ of the CFT and by tracing over the degrees of freedom in the complement of region $A$. We restrict ourselves to the case where $A$ is a single interval and $|s\rangle$ is an eigenstate of the CFT Hamiltonian, so the time evolution of the problem is trivial. Even for these simple situations, the $\mathrm{EE}$ in general depends on all details of the CFT. Thus, in order to have an analytic approach and match the supergravity result, we focus on the short interval limit as discussed above.

As usual [1], we start by considering $n$ independent copies of the original CFT and then insert at the endpoints of the interval $A$ twist fields $\mathcal{T}_{ \pm n}$ that introduce a monodromy which identifies two consecutive sheets. For instance if $T_{j}(z)$ is the (holomorphic part of the) stress energy tensor defined on the $j$ th copy, then $T_{j} \rightarrow T_{j \pm 1}$ when it goes around the operator $\mathcal{T}_{ \pm n}$. The same monodromy holds also for the antiholomorphic fields. Properties and correlators of twist fields have been extensively discussed in several contexts; for a discussion inspired by AdS/CFT see [34-36]. Even the simplest correlators in the presence of twist fields are defined on a complicated world sheet that is obtained by gluing at the positions of the $\mathcal{T}$ s the different sheets where each copy of the CFT is defined. In general the EE of the interval $A=[0, l]$ in the state $|s\rangle$ is given by ${ }^{5}$

$$
S_{A}^{(s)}=-\left.\frac{\partial}{\partial n} S_{n}^{(s)}\right|_{n=1}, \quad S_{n}^{(s)}=\left\langle s\left|\mathcal{T}_{n}(z, \bar{z}) \mathcal{T}_{-n}(w, \bar{w})\right| s\right\rangle,
$$

where

$$
z-w=i \frac{l}{R}
$$

\footnotetext{
${ }^{5}$ Rényi entropies $S_{n}^{(s) R}$ are related to $S_{n}^{(s)}$ as

$$
S_{n}^{(s) R}=\frac{1}{1-n} \log S_{n}^{(s)} .
$$
}


is a point in the complex plane at a distance $l$ from the origin, in a constant time slice.

In the limit of small intervals, all the information we need about the branched world sheet is encoded in the operator product expansion (OPE) of the product of two twist fields $[11,37,38]$,

$$
\begin{aligned}
& \mathcal{T}_{n}(z, \bar{z}) \mathcal{T}_{-n}(w, \bar{w}) \\
& \quad=|z-w|^{-4 \Delta}\left(1+\sum_{K}(z-w)^{\Delta_{K}}(\bar{z}-\bar{w})^{\bar{\Delta}_{K}} \mathcal{D}_{K} \mathcal{O}_{K}(0)\right) .
\end{aligned}
$$

Here $\mathcal{O}_{K}$ is a set of quasiprimary operators living in the $n$th product $\mathcal{C}^{n}$ of the original $\mathrm{CFT} \mathcal{C}, \mathcal{D}_{K}$ is the OPE coefficient for the operator $\mathcal{O}_{K}, \Delta_{K}$ and $\bar{\Delta}_{K}$ are the dimensions of the holomorphic and the antiholomorphic parts of $\mathcal{O}_{K}$, and $\Delta=\bar{\Delta}=c / 24(n-1 / n)$ is the conformal dimension of $\mathcal{T}_{n}$. It is important for our purposes that the operators appearing in the OPE are untwisted, i.e. they are products of operators $O^{(j)}$ defined in the original CFT on each sheet separately: $\mathcal{O}=O^{(1)} \otimes \ldots \otimes O^{(n)}$. By following the standard treatment used in the setup of two disjoint intervals $[11,37,38]$ we order the contributions to the OPE (4.4) according to the number of constituents that are nontrivial $\left(O^{(j)} \neq 1\right)$. So we can write

$$
\begin{aligned}
& \mathcal{T}_{n}(z, \bar{z}) \mathcal{T}_{-n}(w, \bar{w}) \\
& =|z-w|^{-4 \Delta}\left[1+\sum_{K, j}(z-w)^{\Delta_{K}}(\bar{z}-\bar{w})^{\bar{\Delta}_{K}} d_{K}^{(j)} O_{K}^{(j)}\right. \\
& \quad+\sum_{K, L, j_{1} \neq j_{2}}(z-w)^{\Delta_{K}+\Delta_{L}}(\bar{z}-\bar{w})^{\bar{\Delta}_{K}+\bar{\Delta}_{L}} d_{K L}^{\left(j_{1}, j_{2}\right)} \\
& \left.\quad \times O_{K}^{\left(j_{1}\right)} \otimes O_{L}^{\left(j_{2}\right)}+\cdots\right] .
\end{aligned}
$$

Clearly in the small $|z|$ limit we can focus on the operators with the smallest dimension. In any conformal block the operator with the smallest dimension is of course the primary operator. When only the operator on the $j$ th sheet $O_{K}^{(j)}$ is nontrivial, the OPE coefficient $\mathcal{D}_{K}$ is indicated as $d_{K}^{(j)}$. This coefficient is proportional to the one-point function of $O_{K}^{(j)}$ on the $n$th sheeted surface, which can be mapped to the complex plane by an $n$th valued conformal map [11]; since primary operators transform homogeneously under conformal transformations, the corresponding $d_{K}^{(j)}$ s are proportional to the one-point functions on the complex plane that vanish for nontrivial primaries. Thus $d_{K}^{(j)}=0$ when $O_{K}^{(j)}$ is primary. Nonprimary operators can instead have $d_{K}^{(j)} \neq 0$, as it is the case for the stress energy tensor, which is the nonprimary with the smallest dimension. However the states we consider are RR ground states, and in these states the stress energy tensor has a vanishing vacuum expectation value (VEV), as it was verified in [39].

Hence, the first nontrivial contribution which is of interest to us comes from the second term in (4.5), with nontrivial operators on two distinct copies of $\mathcal{C}$. We can moreover restrict the two operators to be primaries, as this will give the dominant contribution for small $|z|$. In this case the OPE coefficients will be indicated as $d_{K L}^{\left(j_{1}, j_{2}\right)}$ and have a simple general expression $[11,38]$

$$
\begin{aligned}
d_{K L}^{\left(j_{1}, j_{2}\right)}= & \sum_{K^{\prime}, L^{\prime}}\left(\mathcal{N}^{-1}\right)_{K L, K^{\prime} L^{\prime}} \lim _{z \rightarrow \infty}|z|^{4 \Delta} \\
& \times\left\langle 0\left|\mathcal{T}_{n}(z, \bar{z}) O_{K^{\prime}}^{\left(j_{1}\right)} \otimes O_{L^{\prime}}^{\left(j_{2}\right)}(1) \mathcal{T}_{-n}(0)\right| 0\right\rangle \\
= & \sum_{K^{\prime}, L^{\prime}}\left(\mathcal{N}^{-1}\right)_{K L, K^{\prime} L^{\prime}} \mathcal{N}_{K^{\prime} L^{\prime}}\left(\frac{1}{2 i n} \frac{1}{\sin \frac{\pi\left|j_{1}-j_{2}\right|}{n}}\right)^{2 \Delta_{K^{\prime}}} \\
& \times\left(\frac{1}{-2 i n} \frac{1}{\sin \frac{\pi\left|j_{1}-j_{2}\right|}{n}}\right)^{2 \bar{\Delta}_{K^{\prime}}},
\end{aligned}
$$

where $\mathcal{N}_{K L, K^{\prime} L^{\prime}}$ is given by the vacuum two-point function of the operators in $\mathcal{C}^{n}$ (such as $\mathcal{O}=O_{K}^{\left(j_{1}\right)} \otimes O_{L}^{\left(j_{2}\right)}$ ),

$$
\mathcal{N}_{K L, K^{\prime} L^{\prime}}=\left\langle 0\left|O_{K}(1) O_{K^{\prime}}(0)\right| 0\right\rangle\left\langle 0\left|O_{L}(1) O_{L^{\prime}}(0)\right| 0\right\rangle,
$$

while the normalization $\mathcal{N}_{K^{\prime} L^{\prime}}$ is defined by the following correlator in $\mathcal{C}$ :

$$
\mathcal{N}_{K^{\prime} L^{\prime}}=\left\langle 0\left|O_{K^{\prime}}(1) O_{L^{\prime}}(0)\right| 0\right\rangle .
$$

This correlator is nontrivial only when $\Delta_{K^{\prime}}=\Delta_{L^{\prime}}$, which was used to simplify (4.6).

Substituting (4.5) and (4.6) in (4.2), we find

$$
\begin{aligned}
S_{n}^{(s)}= & \frac{1}{l^{4 \Delta}}\left[1+\sum_{K, L}\left(\frac{l}{2 n R}\right)^{2\left(\Delta_{K}+\bar{\Delta}_{K}\right)}\left\langle O_{K L}\right\rangle_{s}\right. \\
& \left.\times \sum_{k=1}^{n-1} \frac{n-k}{\left(\sin \frac{\pi k}{n}\right)^{2\left(\Delta_{K}+\bar{\Delta}_{K}\right)}}\right],
\end{aligned}
$$

where

$$
\left\langle O_{K L}\right\rangle_{s} \equiv \sum_{K^{\prime}, L^{\prime}}\left(\mathcal{N}^{-1}\right)_{K L, K^{\prime} L^{\prime}} \mathcal{N}_{K^{\prime} L^{\prime}}\left\langle s\left|O_{K^{\prime}}\right| s\right\rangle\left\langle s\left|O_{L^{\prime}}\right| s\right\rangle
$$

is given in terms of the VEVs of the primary operators $O_{K}$ in the state $|s\rangle$ computed in one copy of the original CFT $\mathcal{C}$. We have used the fact that $\mathcal{N}_{K L, K^{\prime} L^{\prime}}$ is nontrivial only in the subspaces with $\Delta_{K}=\Delta_{K}{ }^{\prime}$ and $\Delta_{L}=\Delta_{L}{ }^{\prime}$ to replace the $\Delta_{K}{ }^{\prime}$ in (4.6) with $\Delta_{K}$. The factor $n-k$ appearing in the sum 
over $k$ accounts for the number of terms in the sums over $j_{1}$ and $j_{2}$ with $\left|j_{1}-j_{2}\right|=k$.

From now on, we specialize our analysis to the D1-D5 SCFT $\mathcal{C}_{D 1 D 5}$ mentioned in the introduction. In particular the $S U(2) \times S U(2)$ R-symmetry of this superconformal field theory (SCFT) plays an important role in our calculation. The above expression for $S_{n}^{(s)}$ is valid at any point in the moduli space of $\mathcal{C}_{D 1 D 5}$. However, for generic primaries $O_{K}$, both the conformal dimensions $\left(\Delta_{K}, \bar{\Delta}_{K}\right)$ and the VEVs $\left\langle O_{K L}\right\rangle_{s}$ are nonprotected quantities and might depend on the couplings. This is hardly a surprise, and indeed entanglement and Rényi entropies do not enjoy in general any nonrenormalization property. In particular the value of $S_{A}^{(s)}$ derived from (4.9) at the free orbifold point of $\mathcal{C}_{D 1 D 5}$ does not match with the gravity result (3.29). When the coupling is increased towards the regime where classical gravity is valid, most of the primary operators will get higher and higher conformal dimensions, and their contribution to (4.9) will become more and more negligible. Hence to compare with gravity one should keep in (4.9) only the chiral primary operators, whose dimensions are finite in the strong coupling regime. The VEV of a chiral primary $O_{K}$ in a 1/4-BPS state is equal to the three-point correlator in the vacuum of three chiral primary operators (the other two being the operators that generate the BPS state when acting on the vacuum). These correlators are known to be protected $[40,41]$. Hence we can compute the VEVs $\left\langle O_{K}\right\rangle_{s}$ at the free point of the CFT or, holographically, from the gravity solution, and the two results should match. The holographic computation of the VEVs has been done in $[26,39]$. Of course to compare with gravity one should also take the limit of large central charge $c=6 n_{1} n_{5} \gg 1$. As we will show below, with our conventions the one-point functions $\left\langle O_{K}\right\rangle_{s}$ and the normalizations $\mathcal{N}_{K L}$ are proportional to $c$, and the coefficients $\mathcal{N}_{K L, K^{\prime} L^{\prime}}$ are proportional to $c^{2}$. Remembering also that the dimension of the twist fields is linear in $c$, one sees that every term in (4.9) gives a contribution to the EE of order $c$. Our computation has to be contrasted with the computation of $\mathrm{EE}$ for two (or more) small intervals in the vacuum $[3,4,38]$ : in that case one has to take the product of two (or more) copies of the OPE in (4.5) and evaluate their correlator in the vacuum. From (4.5) and (4.6) one can see that in this case the contribution from nontrivial primaries is of order $c^{0}$. Thus the EE for more than one interval in the vacuum at large $c$ is a universal quantity, which receives contributions proportional to $c$ only from the conformal block of the identity.

In the D1-D5 SCFT, the first nontrivial chiral primaries have total dimension $\Delta_{K}+\bar{\Delta}_{K}=1$ and will thus contribute corrections of order $l^{2}$ to the EE: these are precisely the corrections expected from (3.29). For operators with this conformal dimension, the sum over $k$ appearing in (4.9) becomes

$$
\begin{aligned}
\sum_{k=1}^{n-1} \frac{n-k}{\sin ^{2} \frac{\pi k}{n}} & =\frac{n}{2} \sum_{k=1}^{n-1} \frac{1}{\sin ^{2} \frac{\pi k}{n}} \\
& =-2 n^{2} \oint \frac{d z}{2 \pi i} \frac{1}{\left(1-z^{n}\right)\left(z^{2}-2 z+1\right)} \\
& =\frac{n\left(n^{2}-1\right)}{6},
\end{aligned}
$$

where in the last step we rewrote the sum as a standard anticlockwise contour integral over $z=e^{2 \pi i k / n}$ around $z=1$.

The chiral primaries with total dimension 1 that are relevant for our purposes are the holomorphic and antiholomorphic $S U(2) \times S U(2)$ currents $J^{\alpha}$ and $\tilde{J}^{\alpha}$ and the operators of dimension $(1 / 2,1 / 2)$ denoted as $O_{(1) 1}^{(1,1)}$ and $O_{(2)}^{(0,0)}$ in [26] [actually the last two operators form quadruplets which transform as vectors of the $S O(4)$ acting on the $S^{3}$ coordinates]. The VEVs of these operators are related with the gravity parameters $a_{\alpha \pm}, \mathcal{A}_{1 i}$, and $f_{1 i}^{1}$ as [26]

$$
\begin{gathered}
\left\langle J^{\alpha}\right\rangle_{s}=c_{J} a_{\alpha+}, \quad\left\langle\tilde{J}^{\alpha}\right\rangle_{s}=c_{\tilde{J}} a_{\alpha-}, \\
\left\langle O_{(1) 1 i}^{(1,1)}\right\rangle_{s}=c_{O^{(1,1)}} \mathcal{A}_{1 i}, \quad\left\langle O_{(2) i}^{(2,2)}\right\rangle_{s}=c_{O^{(2,2)}} f_{1 i}^{1},
\end{gathered}
$$

where the coefficients $c$ depend on the choice of normalization for the various operators. R-symmetry implies that the nonvanishing two-point functions are

$$
\begin{aligned}
\left\langle 0\left|J^{\alpha}(1) J^{\beta}(0)\right| 0\right\rangle & =\mathcal{N}_{J} \delta^{\alpha \beta}, \\
\left\langle 0\left|\tilde{J}^{\alpha}(1) \tilde{J}^{\beta}(0)\right| 0\right\rangle & =\mathcal{N}_{\tilde{J}} \delta^{\alpha \beta}, \\
\left\langle 0\left|O_{(1) 1 i}^{(1,1)}(1) O_{(1) 1 j}^{(1,1)}(0)\right| 0\right\rangle & =\mathcal{N}_{O^{(1,1)}} \delta_{i j}, \\
\left\langle 0\left|O_{(2) i}^{(2,2)}(1) O_{(2) j}^{(2,2)}(0)\right| 0\right\rangle & =\mathcal{N}_{O^{(2,2)}} \delta_{i j} .
\end{aligned}
$$

Then the EE obtained from (4.2) and (4.9) has the form

$$
\begin{aligned}
S_{A}^{(s)}= & {\left[2 n_{1} n_{5} \log \frac{l}{R}-\frac{l^{2}}{12 R^{2}}\left(\mathcal{N}_{J}^{-1} c_{J}^{2} a_{+}^{2}+\mathcal{N}_{\tilde{J}}^{-1} c_{\tilde{J}}^{2} a_{-}^{2}\right.\right.} \\
& \left.\left.+\mathcal{N}_{O^{(1,1)}}^{-1} c_{O^{(1,1)}}^{2} \mathcal{A}_{1}^{2}+\mathcal{N}_{O^{(2,2)}}^{-1} c_{O^{(2,2)}}^{2} f_{1}^{2}\right)\right],
\end{aligned}
$$

which agrees, in structure, with the gravity result (3.29). To refine the comparison and determine also the numerical coefficients, one needs to know the precise normalization of the various operators. We fix the normalizations by comparison with the particular two-charge geometry which was first considered in Sec. VID of [26], where the corresponding state in the language of the dual CFT was also identified. An explicit representation of this state at the free orbifold point of the CFT was worked out in [42]. It is straightforward to check that this state has nontrivial VEVs for $J^{3}, \tilde{J}^{3}$, and $O_{(1)}^{(1,1)}$, and this enables us to uniquely 
determine the values of $c_{J}, c_{\tilde{J}}, c_{O^{(1,1)}}, \mathcal{N}_{J}, \mathcal{N}_{\tilde{J}}, \mathcal{N}_{O^{(1,1)}}$. The operator $O_{(2)}^{(0,0)}$ is of a qualitatively different nature, because it involves a twist field of the orbifold CFT: we will leave the analysis of states with nontrivial VEVs of this operator to a future investigation, and for the moment restrict to geometries with $f_{1}^{2}=0$.

The values of the parameters $a_{\alpha, \pm}$ and $\mathcal{A}_{1 i}$ for the twocharge geometry under consideration can be read off from Eqs. (3.11) of [42], using the identifications $A \equiv-\frac{\beta+\omega}{\sqrt{2}}$, $\mathcal{A} \equiv Z_{4}$. After expanding these quantities for large $r$ and comparing with (3.8), one finds the following nontrivial values:

$$
a_{3+}=-a_{3-}=\frac{R a^{2}}{2 \sqrt{Q_{1} Q_{5}}}, \quad \mathcal{A}_{11}=\frac{R a b}{2 \sqrt{Q_{1} Q_{5}}},
$$

where the radius $R$ is related with other parameters of the geometry by

$$
R=\sqrt{\frac{Q_{1} Q_{5}}{a^{2}+\frac{b^{2}}{2}}} .
$$

The relevant CFT operators are given by ${ }^{6}$

$$
\begin{aligned}
J^{3} & =\sum_{\ell} \frac{1}{2}\left(\chi_{\ell}^{1} \bar{\chi}_{\ell}^{1}+\chi_{\ell}^{2} \bar{\chi}_{\ell}^{2}\right), \quad \tilde{J}^{3}=\sum_{\ell} \frac{1}{2}\left(\tilde{\chi}_{\ell}^{1} \tilde{\bar{\chi}}_{\ell}^{1}+\tilde{\chi}_{\ell}^{2} \tilde{\bar{\chi}}_{\ell}^{2}\right), \\
O & \equiv O_{(1) 11}^{(1,1)}+i O_{(1) 12}^{(1,1)}=\frac{1}{\sqrt{2}} \sum_{\ell}\left(\bar{\chi}_{\ell}^{1} \tilde{\bar{\chi}}_{\ell}^{2}-\bar{\chi}_{\ell}^{2} \tilde{\bar{\chi}}_{\ell}^{1}\right),
\end{aligned}
$$

where the sum over $\ell$ runs over the $n_{1} n_{5}$ copies of the orbifold CFT. From these expressions it is immediate to compute the normalizations

$$
\mathcal{N}_{J}=\mathcal{N}_{\tilde{J}}=\frac{n_{1} n_{5}}{2}, \quad \mathcal{N}_{\mathcal{O}^{(1,1)}}=n_{1} n_{5}
$$

The state dual to this geometry is

$$
|s\rangle=\sum_{k=0}^{n_{1} n_{5}} C_{k} \frac{O^{k}}{k !}\left|n_{1} n_{5} / 2\right\rangle
$$

where $\left|n_{1} n_{5} / 2\right\rangle$ is the unique two-charge state with $J^{3}=\tilde{J}^{3}=n_{1} n_{5} / 2$, and the coefficients $C_{k}$ are

$$
C_{k}=\left(\frac{R a}{\sqrt{Q_{1} Q_{5}}}\right)^{n_{1} n_{5}-k}\left(\frac{R b}{2 \sqrt{Q_{1} Q_{5}}}\right)^{k} .
$$

\footnotetext{
${ }^{6}$ The $\chi$ s are free fermionic fields and we follow the notation of [42].
}

One can thus explicitly compute the VEVs of the relevant operators on this state:

$$
\left\langle J^{3}\right\rangle_{s}=\left\langle\tilde{J}^{3}\right\rangle_{s}=\frac{n_{1} n_{5}}{2} \frac{R^{2} a^{2}}{Q_{1} Q_{5}}, \quad\langle O\rangle_{s}=n_{1} n_{5} \frac{R^{2} a b}{Q_{1} Q_{5}} .
$$

Comparing these VEVs with (4.12) and (4.15), one finds

$$
c_{J}=c_{\tilde{J}}=n_{1} n_{5} \frac{R}{\sqrt{Q_{1} Q_{5}}}, \quad c_{O^{(1,1)}}=2 n_{1} n_{5} \frac{R}{\sqrt{Q_{1} Q_{5}}} .
$$

Substituting in (4.14) one gets

$$
S_{A}^{(s)}=2 n_{1} n_{5}\left[\log \frac{l}{R}-\frac{l^{2}}{12 Q_{1} Q_{5}}\left(a_{+}^{2}+a_{-}^{2}+2 \mathcal{A}_{1}^{2}\right)\right],
$$

which matches with (3.29), when $f_{1}^{2}=0$.

\section{DISCUSSION}

In this paper we focused on the EE for a single interval in a $1+1$ CFT. It is well known that this quantity depends only on the CFT central charge in the simplest case [1], i.e. when the EE is calculated by using a density matrix obtained starting from the $S L(2, \mathbb{C})$ invariant vacuum of the CFT and tracing over the degrees of freedom outside the interval. Not surprisingly, the situation is more complicated if one starts from a generic eigenstate $|s\rangle$ of the CFT Hamiltonian. In order to discuss analytically the $\operatorname{EE} S^{(s)}$ in these situations, we studied the short interval expansion and showed that already the first subleading term depends both on the details of the CFT and the state used to derive the density matrix.

We focused in particular on the SCFT that is dual (in the AdS/CFT sense) to the D1-D5 system in type IIB string theory. This duality provides a precise setting to carry out the same calculation holographically by working with explicit geometries that solve the supergravity equations. We studied in particular the simplest class of regular geometries that are 1/4-BPS. Even in this very simple case the EE $S^{(s)}$ for a single interval depends on the details of the CFT, including the values of the various moduli. In the strongly coupled regime where supergravity is a good approximation we can compare the holographic result against the CFT expectation. In particular we showed that the holographic VEVs derived in [26,39] are in perfect agreement with the result for the EE obtained from the generalization of the RT/HRT formula proposed in (2.2) that applies to six-dimensional spacetimes asymptotic to $\mathrm{AdS}_{3} \times S^{3}$. We thus verify that the RT/HRT holographic formalism for the computation of EE holds also in the presence of nonuniversal contributions. 
It is interesting to compare our results with those of [12], where the thermodynamics properties of the EE for excited states were first discussed. Since we focus on states that are not a small perturbation of the ground state, the final results are qualitatively different. For instance we have to deal in general with a nontrivial dependence on the coordinates outside the AdS space and so the natural approach is to consider the minimal area of a four-dimensional manifold which extends in the $S^{3}$ directions. This also implies that the relation between the variation of the $\mathrm{EE}$ and the variation of the energy stored in the interval for different states does not follow the standard first law-like formula for small perturbations of the vacuum state [12]. In the case analyzed in this paper, all 1/4-BPS states have the same (zero) energy density while the EE changes. A violation of the first law-like relation for large time-dependent perturbations was also noted in [15].

There are of course several possible generalizations of the analysis presented here that might be interesting to pursue. We expect the generic features of the holographic calculation to be common also to higher dimensional cases, such as the 1/2-BPS geometries of [43] that are dual to states in $\mathcal{N}=4 \mathrm{SYM}$. On the CFT side the EE is not captured by correlators among local operators anymore, but it would still be interesting to study holographically the dependence of the EE on the particular state (geometry) considered. Another application of the approach described here is to use the $\mathrm{EE}$ as an observable characterizing the different microstate geometries that have the same asymptotic charges. It would certainly be interesting to extend our analysis to 1/8-BPS (three-charge) configurations and to large intervals. In the latter case the relevant manifold describing the EE extends deep inside the AdS geometry and will be sensitive to the fine details of the different microstates. However, as seen in this paper, even the first subleading term in the short interval expansion depends on the particular microstate geometry considered. So even this simple case could be used to study the relation between the $\mathrm{EE}$ of generic microstates and the thermal state describing the black hole with the same charges. We hope to clarify at least some of these issues in a future work.

\section{ACKNOWLEDGMENTS}

We thank P. Caputa, V. Jejjala, F. Gliozzi, L. Martucci, K. Skenderis, T. Takayanagi, M. Taylor, E. Tonni, and B. Vercnocke for useful discussions and correspondence at several stages of this project. We also thank J. Simon for very useful comments on the first version of this article. This research is partially supported by STFC (Grant No. ST/J000469/1, String theory, gauge theory, and duality), by the Padova University Grant No. CPDA119349, and by INFN. We would like to thank B. Vercnocke for bringing [7] to our attention and for an illuminating discussion on related issues [44]. We are very grateful to J. Simon for drawing our attention to the relevance of the covariant HRT prescription in the context of microstate geometries and also for making us notice that there are various inequivalent ways to extend the $\mathrm{AdS}_{3} \times S^{3}$ splitting from the boundary to the interior, as discussed in Sec. II.
[1] P. Calabrese and J. L. Cardy, J. Stat. Mech. (2004) P06002.

[2] S. Ryu and T. Takayanagi, Phys. Rev. Lett. 96, 181602 (2006).

[3] M. Headrick, Phys. Rev. D 82, 126010 (2010).

[4] T. Hartman, arXiv:1303.6955.

[5] T. Faulkner, arXiv:1303.7221.

[6] A. Lewkowycz and J. Maldacena, J. High Energy Phys. 08 (2013) 090.

[7] P. Caputa, V. Jejjala, and H. Soltanpanahi, Phys. Rev. D 89, 046006 (2014).

[8] M. Caraglio and F. Gliozzi, J. High Energy Phys. 11 (2008) 076.

[9] S. Furukawa, V. Pasquier, and J. Shiraishi, Phys. Rev. Lett. 102, 170602 (2009).

[10] P. Calabrese, J. Cardy, and E. Tonni, J. Stat. Mech. (2009) P11001.

[11] P. Calabrese, J. Cardy, and E. Tonni, J. Stat. Mech. (2011) P01021.

[12] J. Bhattacharya, M. Nozaki, T. Takayanagi, and T. Ugajin, Phys. Rev. Lett. 110, 091602 (2013).
[13] M. Nozaki, T. Numasawa, and T. Takayanagi, Phys. Rev. Lett. 112, 111602 (2014).

[14] S. He, T. Numasawa, T. Takayanagi, and K. Watanabe, arXiv:1403.0702.

[15] P. Caputa, M. Nozaki, and T. Takayanagi, arXiv:1405.5946.

[16] A. Strominger and C. Vafa, Phys. Lett. B 379, 99 (1996).

[17] K. Skenderis and M. Taylor, Phys. Rep. 467, 117 (2008).

[18] V. Balasubramanian, J. de Boer, S. El-Showk, and I. Messamah, Classical Quantum Gravity 25, 214004 (2008).

[19] B. D. Chowdhury and A. Virmani (to be published).

[20] S. D. Mathur, Ann. Phys. (Amsterdam) 327, 2760 (2012).

[21] S. D. Mathur, J. Phys. Conf. Ser. 405, 012005 (2012).

[22] I. Bena and N. P. Warner, arXiv:1311.4538.

[23] S. D. Mathur, Fortschr. Phys. 53, 793 (2005).

[24] O. Lunin and S. D. Mathur, Nucl. Phys. B623, 342 (2002).

[25] O. Lunin, J. M. Maldacena, and L. Maoz, arXiv:hep-th/ 0212210

[26] I. Kanitscheider, K. Skenderis, and M. Taylor, J. High Energy Phys. 06 (2007) 056.

[27] V. E. Hubeny, M. Rangamani, and T. Takayanagi, J. High Energy Phys. 07 (2007) 062. 
[28] X. Dong, J. High Energy Phys. 01 (2014) 044.

[29] J. Camps, J. High Energy Phys. 03 (2014) 070.

[30] A. Bhattacharyya and M. Sharma, arXiv:1405.3511.

[31] S. G. Avery, arXiv:1012.0072.

[32] H. Kim, L. Romans, and P. van Nieuwenhuizen, Phys. Rev. D 32, 389 (1985).

[33] K. Skenderis and M. Taylor, J. High Energy Phys. 05 (2006) 057.

[34] A. Jevicki, M. Mihailescu, and S. Ramgoolam, Nucl. Phys. B577, 47 (2000).

[35] O. Lunin and S. D. Mathur, Commun. Math. Phys. 219, 399 (2001).

[36] O. Lunin and S. D. Mathur, Commun. Math. Phys. 227, 385 (2002).
[37] M. Rajabpour and F. Gliozzi, J. Stat. Mech. (2012) P02016.

[38] E. Perlmutter, J. High Energy Phys. 05 (2014) 052.

[39] I. Kanitscheider, K. Skenderis, and M. Taylor, J. High Energy Phys. 04 (2007) 023.

[40] M. Taylor, J. High Energy Phys. 06 (2008) 010.

[41] M. Baggio, J. de Boer, and K. Papadodimas, J. High Energy Phys. 07 (2012) 137.

[42] S. Giusto and R. Russo, J. High Energy Phys. 03 (2014) 007.

[43] H. Lin, O. Lunin, and J. M. Maldacena, J. High Energy Phys. 10 (2004) 025.

[44] D. Anninos and B. Vercnocke (to be published). 